\title{
ASTROMETRIC MONITORING OF THE HR 8799 PLANETS: ORBIT CONSTRAINTS FROM SELF-CONSISTENT MEASUREMENTS
}

\author{
Q. M. Konopacky ${ }^{1}$, C. Marois $^{2}$, B. A. Macintosh ${ }^{3}$, R. Galicher ${ }^{4,5}$, T. S. Barman ${ }^{6}$, S. A. Metchev ${ }^{7,8}$, and B. Zuckerman ${ }^{9}$ \\ ${ }^{1}$ Center for Astrophysics and Space Sciences, University of California, San Diego, La Jolla, CA 92093, USA; qkonopacky@ ucsd.edu \\ ${ }^{2}$ National Research Council Canada, Dominion Astrophysical Observatory, Victoria, BC V9E 2E7, Canada; christian.marois@ nrc-cnrc.gc.ca \\ ${ }^{3}$ Kavli Institute for Particle Astrophysics and Cosmology, Stanford University, Stanford, CA 94305, USA; bmacintosh@stanford.edu \\ ${ }^{4}$ LESIA, Observatoire de Paris, CNRS, Université Paris Diderot, Université Pierre et Marie Curie, 5 place Jules Janssen, \\ F-92190 Meudon, France; raphael.galicher@obspm.fr \\ ${ }^{5}$ Groupement d'intérêt Scientifique PHASE (Partenariat Haute résolution Angulaire Sol Espace) between ONERA, \\ Observatoire de Paris, CNRS and Université Paris Diderot, France \\ ${ }^{6}$ Lunar and Planetary Lab, University of Arizona, Tucson, AZ 85721, USA; barman@lpl.arizona.edu
${ }^{7}$ Department of Physics and Astronomy, Centre for Planetary Science and Exploration, The University of Western Ontario, \\ London, ON N6A 3K7, Canada; smetchev@uwo.ca \\ ${ }^{8}$ Department of Physics and Astronomy, Stony Brook University, Stony Brook, NY 11794-3800, USA \\ ${ }^{9}$ Department of Physics and Astronomy, University of California, Los Angeles, Los Angeles, CA 90095, USA; ben@astro.ucla.edu \\ Received 2016 March 9; accepted 2016 April 27; published 2016 July 12
}

\begin{abstract}
We present new astrometric measurements from our ongoing monitoring campaign of the HR 8799 directly imaged planetary system. These new data points were obtained with NIRC2 on the W.M. Keck II $10 \mathrm{~m}$ telescope between 2009 and 2014. In addition, we present updated astrometry from previously published observations in 2007 and 2008. All data were reduced using the SOSIE algorithm, which accounts for systematic biases present in previously published observations. This allows us to construct a self-consistent data set derived entirely from NIRC2 data alone. From this data set, we detect acceleration for two of the planets (HR 8799b and e) at $>3 \sigma$. We also assess possible orbital parameters for each of the four planets independently. We find no statistically significant difference in the allowed inclinations of the planets. Fitting the astrometry while forcing coplanarity also returns $\chi^{2}$ consistent to within $1 \sigma$ of the best fit values, suggesting that if inclination offsets of $\lesssim 20^{\circ}$ are present, they are not detectable with current data. Our orbital fits also favor low eccentricities, consistent with predictions from dynamical modeling. We also find period distributions consistent to within $1 \sigma$ with a 1:2:4:8 resonance between all planets. This analysis demonstrates the importance of minimizing astrometric systematics when fitting for solutions to highly undersampled orbits.
\end{abstract}

Key words: astrometry - instrumentation: adaptive optics - planetary systems - stars: individual (HR 8799) techniques: image processing

\section{INTRODUCTION}

Direct imaging offers a powerful tool for the discovery and characterization of Jovian exoplanets. The currently known directly imaged planets are generally massive $\left(\sim 2-10 M_{J}\right)$, residing at wide separations from their host stars $(\sim 10-200 \mathrm{AU}$, e.g., Kalas et al. 2008; Marois et al. 2008, 2010b; Lagrange et al. 2009; Kuzuhara et al. 2013; Rameau et al. 2013; Macintosh et al. 2015). Formation theories do not naturally predict the existence of all directly imaged planets, leading to speculation that dynamical interactions, such as scattering or migration, shortly after the formation of these objects plays a key role in generating their currently observed configuration (e.g., Crida et al. 2009; Veras et al. 2009). In order to assess the dynamical history of these objects, empirical constraints on their current orbital parameters are required.

Given the wide separations of these planets, their predicted orbital periods are tens to hundered of years. This means that while there is some hope of obtaining full orbital phase coverage for the shortest period systems (for example, $\beta$ Pictoris b; Chauvin et al. 2012; Millar-Blanchaer et al. 2015), generally we must be content with fractional orbit coverage. In order to make the most of partial orbital information, precise relative astrometry between the host star and the planet is essential. Such data has been shown to yield useful dynamical constraints in other astronomical contexts (e.g., Duchêne et al. 2006; Lu et al. 2009), and can likely do the same for imaged planets.

The HR 8799 planetary system offers one of the most interesting laboratories for measuring dynamics in a directly imaged system. With four imaged planets (HR 8799b, c, d, and e) ranging in projected separation from $\sim 15$ to $70 \mathrm{AU}$ (Marois et al. 2008, 2010b), the system presents the opportunity to empirically measure orbits and assess the fidelity of those orbit predictions using multiplanet dynamical simulations. Since their original discovery with the W.M. Keck telescope and the Gemini North telescope, the planets in the system system have now been observed by 13 independent high-contrast imaging systems and telescopes, offering a complex, multiwavelength data set spanning 16 years (e.g., Fukagawa et al. 2009; Lafrenière et al. 2009; Metchev et al. 2009; Hinz et al. 2010; Serabyn et al. 2010; Bergfors et al. 2011; Soummer et al. 2011; Skemer et al. 2012; Currie et al. 2014; Ingraham et al. 2014; Pueyo et al. 2015; Rajan et al. 2015; Zurlo et al. 2015).

In exploring the dynamical stability of HR 8799, Fabrycky \& Murray-Clay (2010) were the first to point out that a multiple mean motion Laplace resonance was essential to the long-term stability of the system given the high estimated masses of the planets ( 4-10 $\left.M_{\text {Jup }}\right)$. Similarly, Reidemeister et al. (2009) and Moro-Martín et al. (2010) found that a 1:2:4 resonance was necessary for stability, in addition to a non-face-on orbital inclination for a three-planet system. With the addition of HR 
8799e, Marois et al. (2010b) found that the masses of the planets are likely $\lesssim 7 M_{\text {Jup }}$ based on the stable solutions of Fabrycky \& Murray-Clay (2010) for a three-planet HR 8799 and a younger system age (Zuckerman et al. 2011). In a largescale simulation, Sudol \& Haghighipour (2012) similarly determined that the masses must be $\lesssim 10 M_{\text {Jup }}$. More recently, Goździewski \& Migaszewski (2014) demonstrated that the four HR 8799 planets could have migrated into their current configuration shortly after formation, finding final orbital configurations that are consistent with published astrometry given an inclination of $25^{\circ}$. They also predict the location of a putative additional interior planet given stability requirements (either $\sim 7.5$ or $\sim 9.5 \mathrm{AU}$ ).

Several authors have used existing and new relative astrometric measurements, which typically have a precision of 5-10 milliarcseconds (mas), to empirically constrain the possible orbits of the four HR 8799 planets. In many cases, they have used the results of the dynamical simulations described previously as a starting point for orbit fitting. For example, in their recovery of HR $8799 \mathrm{~b}$, c, and d in archival Hubble Space Telescope (HST) data from 1998, Soummer et al. (2011) tested the 1:2:4 resonance hypothesis, assuming coplanarity, to place constraints on the system inclination and eccentricity. Similarly, Currie et al. (2012) included archival data from Keck to assess the eccentricities and inclinations for the system assuming a 1:2:4 period ratio, finding that face-on orbits did not provide solutions consistent with these periods. They also found that HR 8799d appeared to be non-coplanar with HR 8799b and c. More recently, Esposito et al. (2013) and Maire et al. (2015) have added astrometric data points from the Large Binocular Telescope to constrain possible orbits. They find consistency with the 1:2:4:8 mean motion resonance, but also inclination offsets for HR 8799d. A similar analysis was recently performed by Zurlo et al. (2015) using new data from SPHERE on the Very Large Telescope (VLT). In looking at possible orbits consistent with all available astrometry, Zurlo et al. (2015) conclude that HR $8799 d$ and e may be in a 1:2 or $2: 3$ resonance rather than a possible $2: 5$ resonance. With the addition of an astrometric data point in 2012 from Project 1640, Pueyo et al. (2015) make no assumptions about the orbital properties, instead fitting for all orbital parameters with generously large priors. They also find that HR 8799d has an inclination differing from the other planets. Pueyo et al. (2015) also assert that the masses must be below $13 M_{\text {Jup }}$, consistent with previous dynamical analyses. All of these works combine astrometry from multiple telescopes and instruments, with a large fraction of astrometric data coming from data taken with Keck.

In this paper, we present new and updated astrometric measurements for the four HR 8799 planets obtained with Keck II. By removing systematic biases in our astrometry, we are able to construct distributions of potential orbits from a fully self-consistent data set. In Section 2, we describe our data and our improved reduction methods. In Section 3, we describe our method for orbit fitting and summarize the orbit parameters allowed with our new astrometry and our detection of acceleration in two of the planets. In Section 4 we discuss our findings in the context of other analyses and describe future measurements that could further elucidate the dynamical history of this exoplanetary system.
Table 1

Log of NIRC2 Observations

\begin{tabular}{|c|c|c|c|c|}
\hline $\begin{array}{l}\text { Date } \\
\text { (UT) }\end{array}$ & Filter & $\begin{array}{l}\text { Coronagraph } \\
\text { Size (mas) }\end{array}$ & $\begin{array}{l}\text { Total Int. } \\
\text { Time (s) }\end{array}$ & $\begin{array}{l}\text { Update } \\
\text { or New? }\end{array}$ \\
\hline 2007 Aug 02 & $H$ & 1000 & 3660 & Update $^{\mathrm{a}}$ \\
\hline 2007 Oct 25 & $\mathrm{CH}_{4} \mathrm{~S}$ & none & 2340 & Update \\
\hline 2008 Sep 18 & $K \mathrm{~s}$ & 800 & 1160 & Update \\
\hline 2009 Jul 30 & $K \mathrm{p}$ & 600 & 2800 & New \\
\hline 2009 Aug 01 & $L \mathrm{p}$ & none & 3200 & New \\
\hline 2009 Nov 01 & $L \mathrm{p}$ & none & 2250 & New \\
\hline 2010 Jul 13 & $K \mathrm{~s}$ & 400 & 1460 & New \\
\hline 2010 Oct 30 & $L \mathrm{p}$ & none & 5900 & New \\
\hline 2011 Jul 21 & $K \mathrm{~s}$ & 400 & 3160 & New \\
\hline 2012 Jul 22 & $K \mathrm{~s}$ & 400 & 3325 & New \\
\hline 2012 Oct 26 & $K \mathrm{~s}$ & 400 & 1940 & New \\
\hline 2013 Oct 16 & $L \mathrm{p}$ & none & 1715 & New \\
\hline 2014 Jul 17 & $L \mathrm{p}$ & none & 4900 & New \\
\hline
\end{tabular}

Note.

a Originally published in Metchev et al. (2009).

\section{ASTROMETRIC DATA AND ANALYSIS}

Imaging data from which relative astrometry can be measured for the HR 8799 system now spans 16 years. Here we report on new epochs of imaging taken between 2009 and 2014, and updated analysis of these data that has led to improvements in our astrometric measurements and uncertainties.

\subsection{New Imaging Data}

New data was obtained with the Keck II 10 m telescope with the facility adaptive optics (AO) system (Wizinowich et al. 2006) and the near-infrared camera, NIRC2 (PI K. Matthews). In all observations, $\mathrm{HR}$ 8799A ( $V \sim 6$; Høg et al. 2000) is used as the natural guide star for the AO system. NIRC2 has a plate scale of $9.952 \pm 0.002$ mas pixel $^{-1}$ and columns that are at a PA of $0.252 \pm 0.009$ relative to north (Yelda et al. 2010). Data were taken in both the $K$-short (Ks, $\left.\lambda_{o}=2.146 \mu \mathrm{m}, \Delta \lambda=0.311 \mu \mathrm{m}\right)$ and $L$-prime $(L \mathrm{p}$, $\left.\lambda_{o}=3.776 \mu \mathrm{m}, \Delta \lambda=0.700 \mu \mathrm{m}\right)$ bands. NIRC2 is equipped with a wheel of coronagraphic masks ranging in diameter from 100 to 2000 mas. When observing at $K$-band we used masks (Table 1) but none were used for $L p$ observations.

As described in Marois et al. (2008) and Metchev et al. (2009), the data were obtained such that Angular Differential Imaging (ADI; Marois et al. 2006) processing could be used during reduction. Observations were therefore conducted in vertical angle mode, in which the telescope pupil is fixed on the science camera and the field of view (FOV) slowly rotates with time about the star. Individual frames of $30 \mathrm{~s}$ exposure time are taken as the FOV rotates, ensuring that the PSF of the planets are not overly "smeared." Because HR 8799 passes very close to zenith over Maunakea, observations were generally taken bracketing transit to ensure maximum field rotation. Sky exposures of the same integration time are taken separately by nodding several arcseconds away from the star for the $L \mathrm{p}$ data. For $K \mathrm{~s}$, our reduction method described in Section 2.2 removes sky background. Table 1 lists the date of all observations, the filter, the size of the coronagraphic mask, and the total exposure time of all frames. For completeness, we also list three epochs 
of previously published observations on which we performed new analysis for improved astrometry (see Section 2.2).

\subsection{Data Reduction and Astrometric Measurements}

In the years since the initial publication of Marois et al. (2008), a number of systematic biases due to the method of data collection and reduction algorithms being used to derive the astrometry from Keck have been uncovered and explored. Specifically, these biases are introduced by the original implementation of the LOCI algorithm (Lafrenière et al. 2007) and taking data in the ADI mode (Marois et al. 2006). These are summarized in Marois et al. (2010a) and include image registration error due to imperfect knowledge of the star position, PSF elongation due to FOV rotation, and PSF modification due to self-subtraction. Newer generation algorithms that are also based on least-squares but implemented such that biases are reduced have led to improvements in the derivation of photometry and astrometry (e.g., Marois et al. 2010a, 2014; Amara \& Quanz 2012; Soummer et al. 2012; Fergus et al. 2014; Meshkat et al. 2014; Gomez Gonzalez et al. 2016).

For this work, we use the Speckle-optimized Subtraction for Imaging Exoplanets (SOSIE) algorithm (Marois et al. 2010a) to both reduce new data obtained between 2010 and 2014, and rereduce previously published data from 2007 to $2009 .{ }^{10}$ Briefly, the algorithm first performs the basic reduction of all images (dark and sky subtraction, flat field reduction, bad pixel removal) and corrects for NIRC2 distortion using the solution from Yelda et al. (2010). Next, the images are registered. For coronagraphic data, the star is faintly visible through the occulting spot and can be used for centroiding. For noncorongraphic data, the core of the star is saturated, so crosscorrelation with a reference PSF is used. Then the PSF subtraction is performed using a least-squares algorithm. Finally, the images are rotated such that north is up and the images are combined.

To avoid biasing the astrometry due to the impact of ADI and LOCI processing on the planet PSF from self-subtraction, a forward modeled PSF template is calculated in all optimization sections based on the LOCI parameters used. This PSF is then used to fit for astrometry and photometry for each planet. To derive uncertainties, a model PSF is used to subtract the planet from the image once the position and flux are known. The residual noise in the region surrounding the location of the subtracted planet is calculated. We then perform a Monte Carlo simulation in which we slightly vary the position of the planet center from the derived location, subtract the PSF, and again compute the noise. This allows us to generate a "noise curve" as a function of planet position. In order to calculate the 1 sigma positional uncertainties, we determine the offset that yields an increase in the local noise by a factor of a square root of 2. This is done separately in the $X$ and $Y$ directions, and the uncertainties in each direction are averaged to derive a final positional uncertainty. A similar process is performed for photometry. Further details on our method of derivation of uncertainties is described in Marois et al. (2010a) and Galicher \& Marois (2011).

\footnotetext{
${ }^{10}$ Keck data were taken by our group in 2004 but in non-ADI mode. Because it is non-ADI, only the outer two planets are detected, and the new pipeline would not improve the astrometry. Thus, it was not re-reduced here, but values from Marois et al. (2008) are included in the subsequent analysis.
}

In the course of obtaining our observations, we also noted that an uncertainty was also introduced by the NIRC2 coronagraphic masks. Specifically, while the semi-transparent nature of these masks is useful for obtaining unsaturated centroids for the star, we found a systematic shift in source positions when the focal plane mask is in place versus when it is not in place. Through a series of tests with the Keck AO internal fiber-fed point source, we determined the extent of this offset by marching the source across the NIRC2 FOV. We found that for the most part this is a uniform shift across the FOV - this was by design, as the focal plane mask was given a slight tilt of $2^{\circ} .603 \pm 0^{\circ} .003$ (K. Matthews 2016, private communication). Thus, for relative astrometry, there should be no impact from having the focal plane mask in the optical path. However, for point sources under the coronagraphic spot, the shift was found to be slightly less than outside of the spot (of the order of $\sim 0.1$ pixels), therefore impacting our relative astrometry. The cause for this difference is unknown. To measure this offset on each data set, an iterative "rotation axis" technique was developed to search for the optimal rotation axis that maximizes the signal-to-noise ratio of the planets. Using this, we find that the uncertainty on the star position is now typical of the order of $\sim 2$ mas.

The resulting $X$ and $Y$ positions and uncertainties were converted into arcseconds using the NIRC2 plate scale from Yelda et al. (2010), and the uncertainties in the plate scale and north angle offset are added in quadrature with the positional uncertainties. All values for all four planets are given in Table 2. The final astrometric uncertainties range from 3 to 22 mas.

\section{ORBIT FITTING}

\subsection{The Construction of a Self-consistent Data Set}

Fitting relative astrometric orbits to data on long-period objects, while technically straightforward, can lead to biased results. Fitting relative orbits to a small percentage of a $>50$ year orbit tends to yield a preference for periastron passage close to the epoch during which the data was taken. As a result, artifically high eccentricities tend to also be preferred. Furthermore, systematics between astrometric data taken from different cameras are noticeable and impactful when full phase coverage is obtained-the situation is worse when only a tiny fraction of the orbit has been measured. In the case of directly imaged planets, a further complication is introduced by the choice of algorithm used to enhance the contrast and yield a robust detection of the planet. Each algorithm uses different methods of deriving astrometry, which can further skew resulting orbital parameters. Assessment of the biases introduced by each pipeline, such as those discussed in relation to SOSIE in Marois et al. (2010a), are ongoing (e.g., Amara \& Quanz 2012; Pueyo et al. 2015). Even when all possible biases are accounted for and uncertainties enlarged to attempt to encompass these errors, systematics across multiple data sets remain. As an example, it has been noted by multiple authors that the previously published Keck astrometry on HR 8799 (Marois et al. 2008, 2010b), when combined with other data, yields poor $\chi^{2}$. For instance, fitting orbits to our previous astrometry for HR 8799 b yields a best fit reduced $\chi^{2}$ of 2.5 . The probability of obtaining this value for $\chi^{2}$ under the assumption of Gaussian uncertainties is $0.002 \%$, highlighting the remaining systematics in our data set. Our improvements in 
Table 2

HR 8799 Relative Astrometry

\begin{tabular}{|c|c|c|c|c|c|c|c|c|}
\hline \multirow[b]{2}{*}{ Date (UT) } & \multicolumn{2}{|c|}{ HR $8799 \mathrm{~b}$} & \multicolumn{2}{|c|}{ HR 8799c } & \multicolumn{2}{|c|}{ HR 8799d } & \multicolumn{2}{|c|}{ HR 8799e } \\
\hline & $\Delta x$ (as) & $\Delta y$ (as) & $\Delta x$ (as) & $\Delta y$ (as) & $\Delta x$ (as) & $\Delta y$ (as) & $\Delta x$ (as) & $\Delta y$ (as) \\
\hline $2004 \mathrm{Jul} 14^{\mathrm{a}}$ & $-1.471 \pm 0.006$ & $0.884 \pm 0.006$ & $0.739 \pm 0.006$ & $0.612 \pm 0.006$ & $\mathrm{n} / \mathrm{a}$ & $\mathrm{n} / \mathrm{a}$ & $\mathrm{n} / \mathrm{a}$ & $\mathrm{n} / \mathrm{a}$ \\
\hline 2007 Aug 02 & $-1.504 \pm 0.003$ & $0.837 \pm 0.003$ & $0.683 \pm 0.004$ & $0.671 \pm 0.004$ & $0.179 \pm 0.005^{\mathrm{b}}$ & $-0.588 \pm 0.005^{\mathrm{b}}$ & $\mathrm{n} / \mathrm{a}$ & $\mathrm{n} / \mathrm{a}$ \\
\hline 2007 Oct 25 & $-1.500 \pm 0.007$ & $0.836 \pm 0.007$ & $0.678 \pm 0.007$ & $0.676 \pm 0.007$ & $0.175 \pm 0.010$ & $-0.589 \pm 0.010$ & $\mathrm{n} / \mathrm{a}$ & $\mathrm{n} / \mathrm{a}$ \\
\hline 2008 Sep 18 & $-1.516 \pm 0.004$ & $0.818 \pm 0.004$ & $0.663 \pm 0.003$ & $0.693 \pm 0.003$ & $0.202 \pm 0.004$ & $-0.588 \pm 0.004$ & $\mathrm{n} / \mathrm{a}$ & $\mathrm{n} / \mathrm{a}$ \\
\hline 2009 Jul 30 & $-1.526 \pm 0.004$ & $0.797 \pm 0.004$ & $0.639 \pm 0.004$ & $0.712 \pm 0.004$ & $0.237 \pm 0.003$ & $-0.577 \pm 0.003$ & $0.306 \pm 0.007$ & $-0.211 \pm 0.007$ \\
\hline 2009 Aug $01^{c}$ & $-1.531 \pm 0.007$ & $0.794 \pm 0.007$ & $0.635 \pm 0.009$ & $0.722 \pm 0.009$ & $0.250 \pm 0.007$ & $-0.570 \pm 0.007$ & $0.318 \pm 0.010$ & $-0.195 \pm 0.010$ \\
\hline 2009 Nov 01 & $-1.524 \pm 0.010$ & $0.795 \pm 0.010$ & $0.636 \pm 0.009$ & $0.720 \pm 0.009$ & $0.251 \pm 0.007$ & $-0.573 \pm 0.007$ & $0.310 \pm 0.009$ & $-0.187 \pm 0.009$ \\
\hline $2010 \mathrm{Jul} 13$ & $-1.532 \pm 0.005$ & $0.783 \pm 0.005$ & $0.619 \pm 0.004$ & $0.728 \pm 0.004$ & $0.265 \pm 0.004$ & $-0.576 \pm 0.004$ & $0.323 \pm 0.006$ & $-0.166 \pm 0.006$ \\
\hline 2010 Oct 30 & $-1.535 \pm 0.015$ & $0.766 \pm 0.015$ & $0.607 \pm 0.012$ & $0.744 \pm 0.012$ & $0.296 \pm 0.013$ & $-0.561 \pm 0.013$ & $0.341 \pm 0.016$ & $-0.143 \pm 0.016$ \\
\hline $2011 \mathrm{Jul} 21$ & $-1.541 \pm 0.005$ & $0.762 \pm 0.005$ & $0.595 \pm 0.004$ & $0.747 \pm 0.004$ & $0.303 \pm 0.005$ & $-0.562 \pm 0.005$ & $0.352 \pm 0.008$ & $-0.130 \pm 0.008$ \\
\hline 2012 Jul 22 & $-1.545 \pm 0.005$ & $0.747 \pm 0.005$ & $0.578 \pm 0.005$ & $0.761 \pm 0.005$ & $0.339 \pm 0.005$ & $-0.555 \pm 0.005$ & $0.373 \pm 0.008$ & $-0.084 \pm 0.008$ \\
\hline 2012 Oct 26 & $-1.549 \pm 0.004$ & $0.743 \pm 0.004$ & $0.572 \pm 0.003$ & $0.768 \pm 0.003$ & $0.346 \pm 0.004$ & $-0.548 \pm 0.004$ & $0.370 \pm 0.009$ & $-0.076 \pm 0.009$ \\
\hline 2013 Oct 16 & $-1.545 \pm 0.022$ & $0.724 \pm 0.022$ & $0.542 \pm 0.022$ & $0.784 \pm 0.022$ & $0.382 \pm 0.016$ & $-0.522 \pm 0.016$ & $0.373 \pm 0.013$ & $-0.017 \pm 0.013$ \\
\hline 2014 Jul 17 & $-1.560 \pm 0.013$ & $0.725 \pm 0.013$ & $0.540 \pm 0.013$ & $0.799 \pm 0.013$ & $0.400 \pm 0.011$ & $-0.534 \pm 0.011$ & $0.387 \pm 0.011$ & $0.003 \pm 0.011$ \\
\hline
\end{tabular}

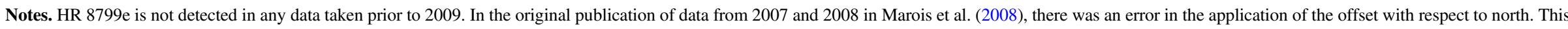
has been remedied here.

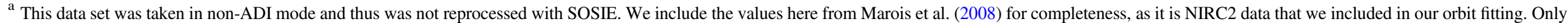
HR $8799 \mathrm{~b}$ and $\mathrm{c}$ are detected in this data set.

${ }^{\mathrm{b}}$ Due to the proximity of HR $8799 \mathrm{~d}$ to the 1000 mas focal plane mask in this epoch, we believe its position is biased. We therefore elected not to include it in orbit fitting.

${ }^{c}$ This epoch was not used for orbit fitting due to the close time sampling to the 2009 July 30 points. We include the astrometry here for completeness. 
reduction and derivation of astrometry improve the overall $\chi^{2}$ fits by factors of $\sim 4-8$, with associated probabilities of Gaussian uncertainties $>90 \%$.

Still, even with our improved reduction, systematics between our data set and data presented in the literature (from multiple cameras and reduction pipelines) remain. The Keck data set is by far the most extensive, spanning $\sim 10$ years for HR $8799 \mathrm{~b}$ and c, $\sim 7$ for HR 8799d, and $\sim 5$ for HR 8799e. Thus, we elect here to estimate the orbital parameters for the system using only this data set. This circumvents most of the remaining systematics, though it does lower the overall time baseline by not including the HST data points from Lafrenière et al. (2009) or Soummer et al. (2011). However, we conducted several sample orbit fits for HR 8799b, c, and d to determine the impact of the HST points on solutions. Due to the large uncertainties on the HST astrometry, all orbital parameters obtained with and without the HST data were consistent to within their uncertainties. The Keck data set, with among the smallest uncertainties obtained, remains the biggest weight in all fits. We also only utilize data reduced through our reduction pipeline, electing not to use the results from Currie et al. (2012, 2014).

\subsection{Acceleration Detections}

With our improved astrometric data, we can first determine whether any orbital curvature (or acceleration) has been detected. In Figure 1, we plot the $x$ (R.A.) and $y$ (decl.) astrometry from Keck used in our analysis as a function of time for all four planets. In order to determine whether acceleration was detected for any of the planets, we fit second-order polynomials of the form given in Equations (3) and (4) of $\mathrm{Lu}$ et al. (2009) to the data shown in Figure 1. These polynomials are shown as lines overplotted on the data in Figure 1. This gives us an estimate of the velocity and acceleration in $x$ and $y$ and an uncertainty. We then converted these into radial and tangential components of velocity and acceleration by a transformation from cartesian to spherical coordinates. Since true orbital acceleration will only have a negative radial component, we consider orbital acceleration "detected" if the measured radial acceleration plus three times its uncertainty is less than zero (or effectively that it is detected to $3 \sigma$ ). This is analogous to the procedure for determining the significance of an acceleration detection described in Lu et al. (2009)

Using this method, we find that we have detected acceleration to $3.4 \sigma$ for HR $8799 \mathrm{~b}\left(-0.35 \pm 0.10 \mathrm{mas} \mathrm{yr}^{-2}\right)$

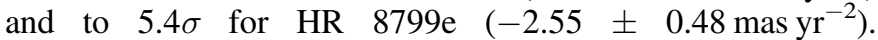
Acceleration is not detected significantly for HR $8799 \mathrm{c}$ $\left(-0.32 \pm 0.21 \mathrm{mas} \mathrm{yr}^{-2}, 2.1 \sigma\right)$ or HR $8799 \mathrm{~d}(-0.65 \pm$ $\left.0.56 \mathrm{mas} \mathrm{yr}^{-2}, 1.2 \sigma\right)$. For all four planets, the (non-physical) tangential acceleration derived from the fits is insignificant $(<0.5 \sigma)$. We anticipate that with continued measurements at a similar cadence to our current observations (one or two times per year), orbital acceleration will be detectable in 2016 for both HR 8799c and HR 8799d using Keck data alone (note that including the HST data point from 1998 would yield an acceleration detection for these planets).

\subsection{Orbital Fitting}

Using the astrometric measurements described above, we now seek to determine the currently allowed astrometric orbital parameters for the HR 8799 planets. Our model for the relative orbit always contains six free parameters: period $(P)$ (related to the semimajor axis (a) through the total system mass), eccentricity $(e)$, time of periapse passage $\left(T_{o}\right)$, inclination $(i)$, position angle of the ascending node $(\Omega)$, and longitude of periapse passage $(\omega)$. We fix the mass of the star to that measured in Baines et al. (2012), 1.516 $M_{\odot}$ for an age of $\sim 30 \mathrm{Myr}$, and increase the uncertainties on this value from the published numbers to $\pm 0.15 M_{\odot}$ (or $10 \%$ of the mass) to account for any possible additional uncertainties in the evolutionary models used to derive the mass. In order to convert from on-sky to physical units, we use the distance to the star measured by van Leeuwen (2007) of $39.4 \pm 1.1 \mathrm{pc}$.

Initial orbital parameter ranges are first estimated by mapping $\chi^{2}$ through a Monte Carlo minimization routine described in Ghez et al. (2008). All orbital parameters are sampled from uniform distributions except for inclination, which is sampled from a distribution uniform in $\cos (i)$. Note that because such a small fraction of the orbit has been mapped for these planets ( $\sim 3 \%$ for HR $8799 \mathrm{~b}, \sim 6 \%$ for HR $8799 \mathrm{c}$, $\sim 12 \%$ for HR $8799 \mathrm{~d}$, and $\sim 6 \%$ for HR $8799 \mathrm{e}$ ), the "best fitting" orbit is not strictly meaningful in its own right. However, the value of reduced $\chi^{2}$ we obtain for the best fits is a gauge of the fidelity of our astrometry and error bars, and a check for systematics remaining in our Keck-only data set. For our choice of data, the best fit reduced $\chi^{2}$ values are $0.36,0.29$, 0.52 , and 0.44 for HR $8799 \mathrm{~b}, \mathrm{c}, \mathrm{d}$, and e, respectively, implying that our uncertainties are in fact slightly overestimated. This is likely due to our conservative approach in assigning an uncertainties, including assumptions about the star centroid and using our Monte Carlo simulation to derive the uncertainties in the planet position. However, it also shows that our method is likely incorporating any remaining systematic uncertainties and validates the choice of using a single consistently reduced data set. As an example of the quality of the fits, we plot loweccentricity solutions that are consistent with the astrometry in Figure 2. Positional residuals between these fits and the astrometry are shown in the insets for each planet. All data points are consistent with these fits to within their $1 \sigma$ uncertainties.

Once we broadly determined the range of allowed parameters, we mapped their probability distributions using a Monte Carlo simulation. First, 100,000 artificial data sets are generated to match the observed data set in number of points, where the value of each point (including the distance and mass of the star) is assigned by randomly drawing from a Gaussian distribution centered on the best fit value with a width corresponding to the uncertainty on that value. Each of these artificial data sets is then fit with an orbit model as described above. From these trials, we saved the best fit model. Our orbital analysis approach is somewhat different from other works, relying on a more standard Monte Carlo implementation than on methods like MCMC. The analysis performed here is computationally intensive, taking anywhere from a factor of 1.5-2.5 times longer to run on an equivalent data set that converges, but also avoids potential issues such as getting stuck in local minima, forcibly exploring parameter space. Methods that are less likely to have issues with complex distributions are preferable in situations such as ours where this could be concerned, in spite of the computational expense. Given proper implementation and exploration of parameter space, the results from the two algorithmic implementations should return equivalent results and uncertainties (e.g., De Rosa et al. 2015). 

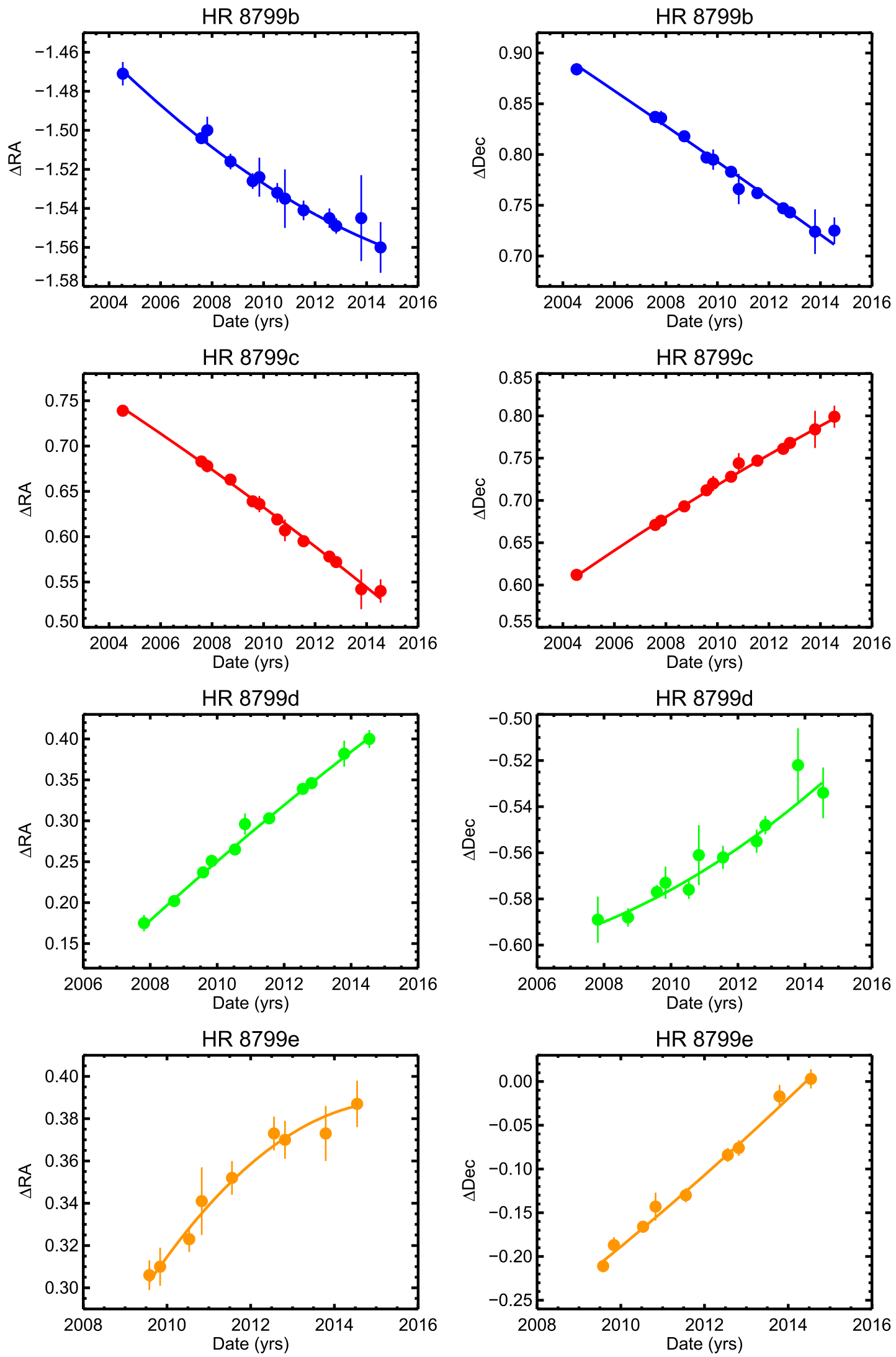

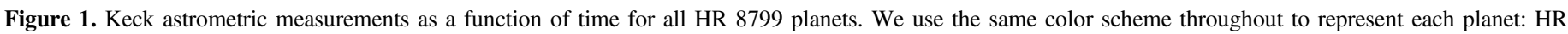

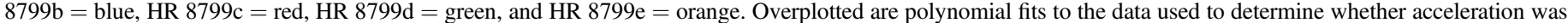

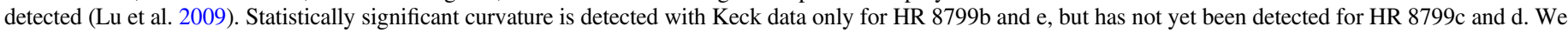
anticipate that with continued monitoring and similar error bars, we will detect acceleration using Keck data in 2016 for HR $8799 \mathrm{c}$ and d. 


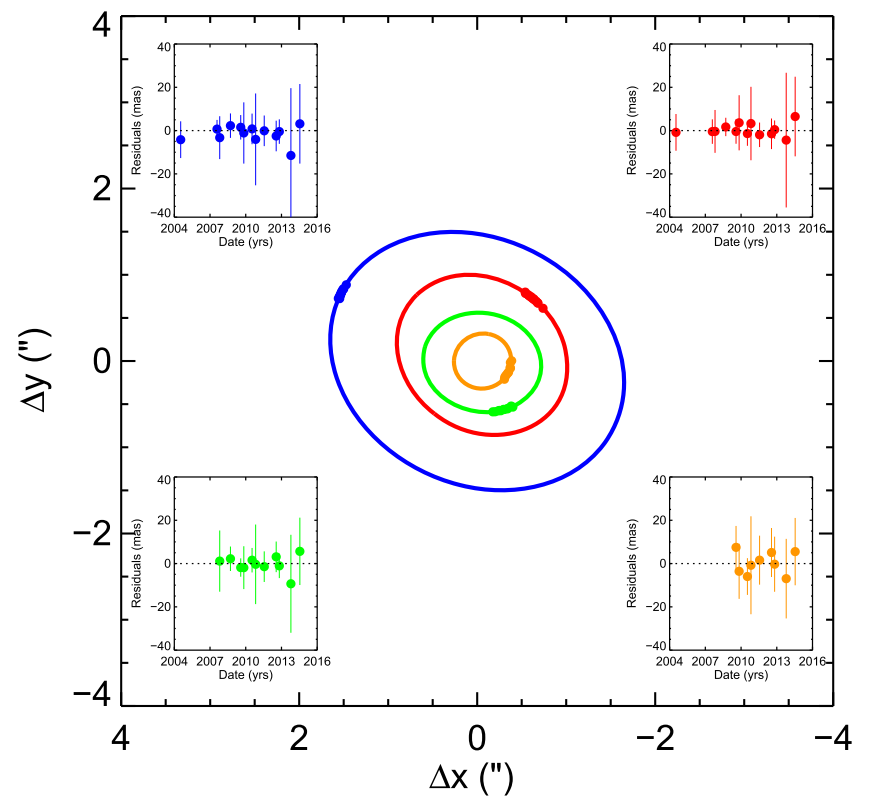

Figure 2. Sample low-eccentricity orbits that are consistent with our astrometry. Each orbit shown is within $1 \sigma$ of the best fit solution. The side panels show the size of the residuals to each of these fits. All residuals are consistent with zero to within our uncertainties. For all four planets, our best fit reduced $\chi^{2}$ is less than 1 , suggesting that our uncertainties are slightly overestimated. This is likely due to our conservative approach to uncertainty assignment for both the positions of the planets and the positions of the central star.

For HR 8799e, we also computed the best fit obtained after an additional weighting for the "likelihood" of a given orbit. As discussed in Section 3.1, highly undersampled orbits are often "best fit" by solutions that are biased toward high eccentricities and $T_{o}$ near the time the data is taken, even when astrometric systematics are minimized. This bias impacts the HR 8799e simulations, where the phase coverage is the smallest and the astrometric uncertainties the greatest. The difference between the distributions for the other planets whether weighted or not is small. We therefore applied a correction factor to the HR 8799e fits, which we calculated by dividing the time baseline of our observations by the average time the planet would spend in another portion of its orbit with comparable arclength, or distance traveled. We multiplied each $\chi^{2}$ by this correction factor, and then saved the best fit after applying this correction. In order to verify that this correction factor was appropriate, we tested this process on simulated astrometry sampled from a notional orbit for HR $8799 \mathrm{e}$ that had an eccentricity of $\sim 0.05$ and went through periastron passage in 1985 . We assigned this astrometry equivalent error bars to our actual data. In this test, we found that when we did not weight our $\chi^{2}$ values, $25 \%$ of the solutions had $T_{o}$ coincident with the time the data was taken, with $75 \%$ within \pm 5 years of the time the data was taken. With weighting, this fell to $0.3 \%$ of solutions with $T_{o}$ during the time the data was taken and $1 \%$ within five years of the data. In addition, without weighting, $64 \%$ of solutions yielded eccentricity $>0.5$, whereas $0.2 \%$ of weighted solutions had eccentricities this high. Thus, we believe that in the case of HR $8799 \mathrm{e}$, the distribution of parameters for weighted orbits has a much greater likelihood of encompassing the true orbital parameters. Note that the impact of weighting on solutions where HR $8799 \mathrm{e}$ is currently at apoastron with a high eccentricity and thus short orbital period are minimally impacted by this weighting, as the probability of observing an eccentric planet at apoastron is high (though these solutions are also unlikely; see Section 3.4).

In order to verify that no data points in our Keck data set given in Table 2 were significantly impacting our resulting orbital parameters, we performed the same fitting routine with each data point individually removed. For HR 8799c and e, the exclusion of any data point has no impact on the parameter ranges. For HR $8799 \mathrm{~b}$ and $\mathrm{d}$, the removal of the 2008 data point has a modest impact on the range of periods (and therefore SMA), increasing the upper limit on periods by about $10 \%$ in both cases. The same is true for HR $8799 \mathrm{~b}$ in the case of the 2004 data point. In the case of the 2008 data, the points are the only ones for that year and have small error bars. For 2004, the error bars are larger but the data point is unique in time sampling. We also see with the removal of the 2004 data point that the increase in allowed periods corresponds to a few degree increase in the range of inclinations for HR 8799b. Still, given that the constraints on all parameters are quite broad using the full data set, we assert that the impact of any one data point on our fits is very modest.

We note that our goal is to obtain all orbital parameters currently allowed by our astrometry. While other authors have focused on solutions based on stability criterion (e.g., Soummer et al. 2011; Maire et al. 2015; Zurlo et al. 2015), we were interested in what regions of parameter space could be ruled out by the self-consistent data set alone assuming Keplerian orbits. Future work will include assessing the stability of orbits allowed from the Keck data.

\subsection{Allowed Orbital Parameters}

To demonstrate the allowed $X-Y$ phase space of orbits by the astrometric data, we plot in Figure 3 the allowed orbits for each planet (converted to AU using a distance of $39.4 \mathrm{pc}$ ). The lefthand panel demonstrates all phase space, while the right-hand panel shows only the two-dimensional projection of orbits that do not cross the Hill radius of another planet.

The full distributions of orbital parameters are shown in Figures 4 through 7 . For all planets, we present both "weighted" and "unweighted" solutions, though they only differ significantly for HR 8799e.

The strongest orbital constraints are found for HR $8799 \mathrm{~b}$ (Figure 4). Strong eccentricity upper limits can be placed at $<0.3$. Generally face-on orbits are not allowed, and we find a preferred inclination of $38^{\circ} \pm 7^{\circ}$. For the other orbital plane parameter, argument of the ascending node $(\Omega)$, we find a peak near $\sim 65^{\circ}$, with a second set of solutions near $\sim 150^{\circ}$. The latter appear to be slightly more correlated with higher eccentricity solutions. The peak of the period distribution is at $\sim 470$ years, which implies a peak SMA of $\sim 70 \mathrm{AU}$. The other two parameters are largely unconstrained.

For HR 8799c (Figure 5), we find a peak at fairly low periods and SMAs, with a broad tail to higher values. This corresponds to the tail of eccentricities that are still allowed, with an upper limit of $<0.5$. The peak value for the period is $\sim 185$ years, corresponding to an SMA of $\sim 38 \mathrm{AU}$. The preferred inclination is $37^{\circ} \pm 12^{\circ}$, consistent with the inclination found for HR $8799 \mathrm{~b}$. Few constraints are found for $\omega, \Omega$, or $T_{o}$. A higher number of low-eccentricity solutions do favor an $\Omega$ near $\sim 50^{\circ}$.

For HR 8799d (Figure 6), the lower astrometric precision leads to an essentially flat eccentricity distribution out to 0.6. Like HR 8799c, the period distribution peaks at relatively low 

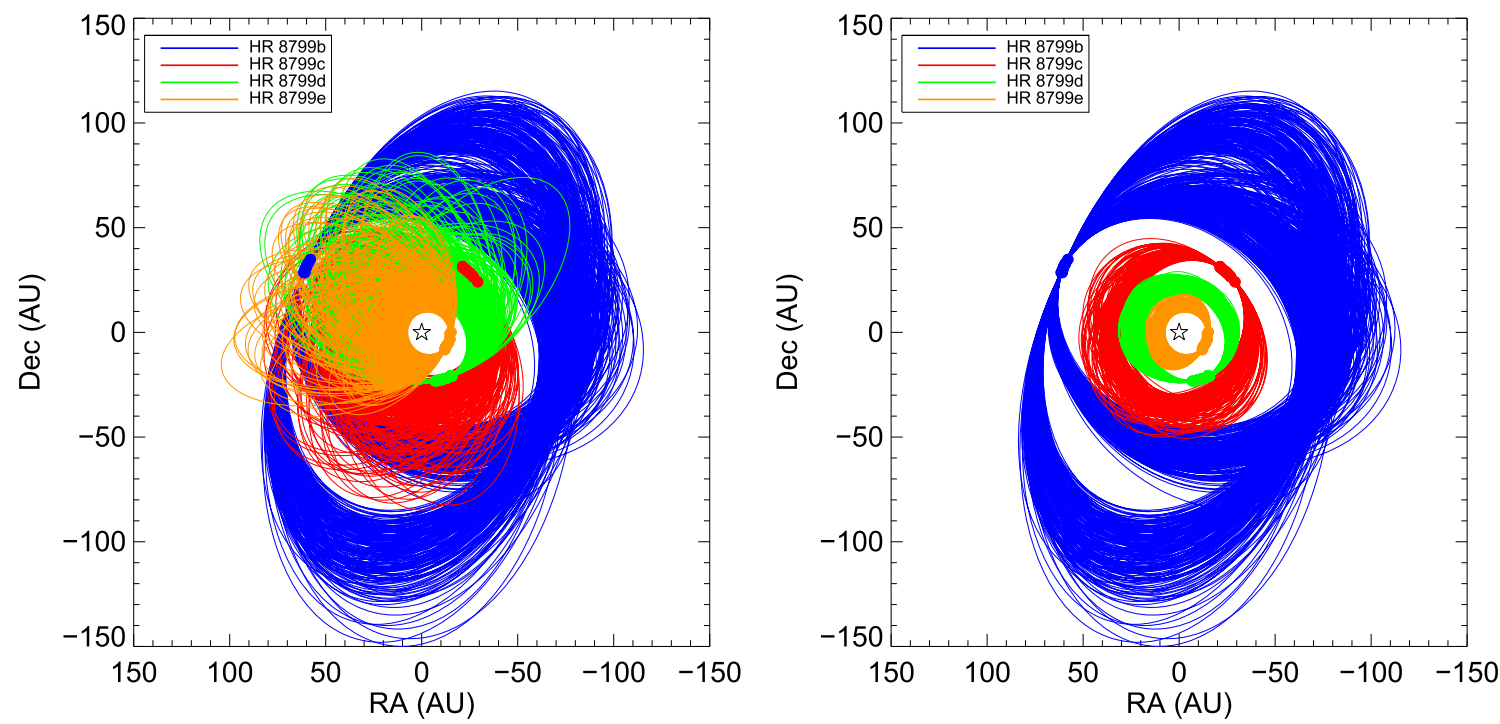

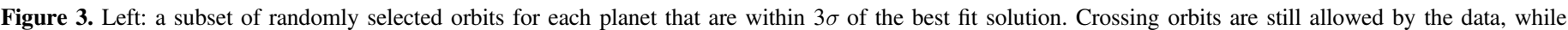

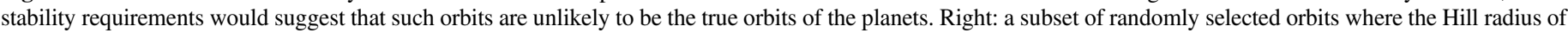

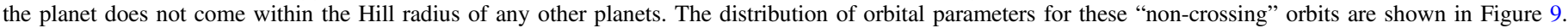

values with a large declining tail toward large periods. The distributions of period and SMA are flatter than for HR 8799c because of the large range of allowed eccentricities. The preferred inclination is $45^{\circ} \pm 8^{\circ}$. This is fully consistent with the values obtained for HR $8799 \mathrm{~b}$ and $\mathrm{c}$. The other parameters are again largely unconstrained. The distribution of $T_{o}$ brackets the epoch where the data was obtained, but spans about 40 years, so does not appear to be strongly biased as we see in the case of HR 8799e.

For HR 8799e (Figure 7), running the simulations unweighted yields an eccentricity distribution skewed toward high eccentricty, with a peak near 0.6 and an upper limit of 0.8 . Weighting significantly reduces the range of allowed eccentricities, with an upper limit of $<0.4$. The preference for eccentricity between 0.1 and 0.2 may possibly be due to the larger astrometric uncertainties rather than an actual elevated eccentricity. Fits in that region tend to favor a face-on configuration, though the distribution extends to $40^{\circ}$ at lower probability. Lower and higher eccentricities tend toward larger inclination, between $20^{\circ}$ and $50^{\circ}$. The inclination predicted for HR $8799 \mathrm{e}$ in the unweighted simulations is $47^{\circ} \pm 6^{\circ}$. As predicted, the unweighted simulations have a peak $T_{o}$ of 2015 , implying that the best fit solutions have periastron passage happening immediately after the last data point was taken. The spread in the peak ranges from 2010 to 2020, thus fully overlapping our data set. Weighting instead shifts the distribution of $T_{o}$ to the mid-1990s, with a peak in 1997. Given the peak in the period distribution for the weighted simulations of $\sim 35$ years and an SMA peak of $\sim 13 \mathrm{AU}$, this range of $T_{o}$ seems fairly reasonable. In the unweighted simulations, the periods and SMAs are fairly flat, extending out to $\sim 400$ years and $\sim 100$ AU, respectively. Weighting also moves $\Omega$ and $\omega$ from fairly peaked at certain values to relatively unconstrained.

To assess the dependence of orbital plane configuration on eccentricity, in Figure 8 we show $1 \sigma$ and $2 \sigma$ contours for the joint probability density functions between eccentricity and inclination and the $1 \sigma$ contours for eccentricity and $\Omega$ (using the weighted fits to HR 8799e). These figures demonstrate that the orbital planes of all four planets are consistent within $<2 \sigma$. They also show the preferred values of inclination and $\Omega$ for low-eccentricity orbits. For $\Omega$, there is overlap between $\sim 50^{\circ}-$ $70^{\circ}$ at low eccentricity. For inclination, there is overlap at $\sim 30^{\circ}$ for low-eccentricity solutions of HR $8799 \mathrm{~b}, \mathrm{c}$, and d at $1 \sigma$, and HR $8799 \mathrm{e}$ at $2 \sigma$.

Although we have not performed any dynamical analyses to assess the possible stability of these orbital solutions, a very rough proxy for viable solutions is to consider only those solutions that do not come within a Hill radius of the other planets, as shown in the left panel of Figure 3. This analysis can be performed using our original simulations (using the weighted version for HR 8799e), which consider each planet independently when fitting to assess the allowed parameter distributions from astrometry, and the nominally preferred masses of the planets $\left(5,7,7\right.$, and $7 M_{\text {Jup }}$ for HR $8799 \mathrm{~b}, \mathrm{c}, \mathrm{d}$, and e, respectively). Additional constraints on the masses of the planets from dynamical arguments requires simultaneous orbit modeling that is beyond the scope of this paper. Nonetheless, we wish to get a rough sense of the distribution of parameters from our simulations that are more likely to be stable solutions. The distributions of orbital parameters for only these solutions are shown in Figure 9. Requiring orbits not to "cross" significantly limits the eccentricity of the inner planets, leading to upper limits of $<0.3$ for all four planets. This naturally leads to lower values for period and SMA. Additionally, the distribution of orbital inclination fully overlaps for all four planets, with values between $30^{\circ}$ and $40^{\circ}$ consistent to within $1 \sigma$. The preferred inclination for HR $8799 \mathrm{~d}$ and e moves to slightly lower values more consistent with the distributions for HR $8799 \mathrm{~b}$ and c. There also seems to be a general preference for $\Omega$ between $40^{\circ}$ and $70^{\circ}$, but larger values are still allowed.

\section{DISCUSSION}

\subsection{Comparison to Previous Analyses}

We have computed distributions of allowed orbital parameters for all of the HR 8799 planets using a data set for which we have attempted to minimize systematic uncertainties in 

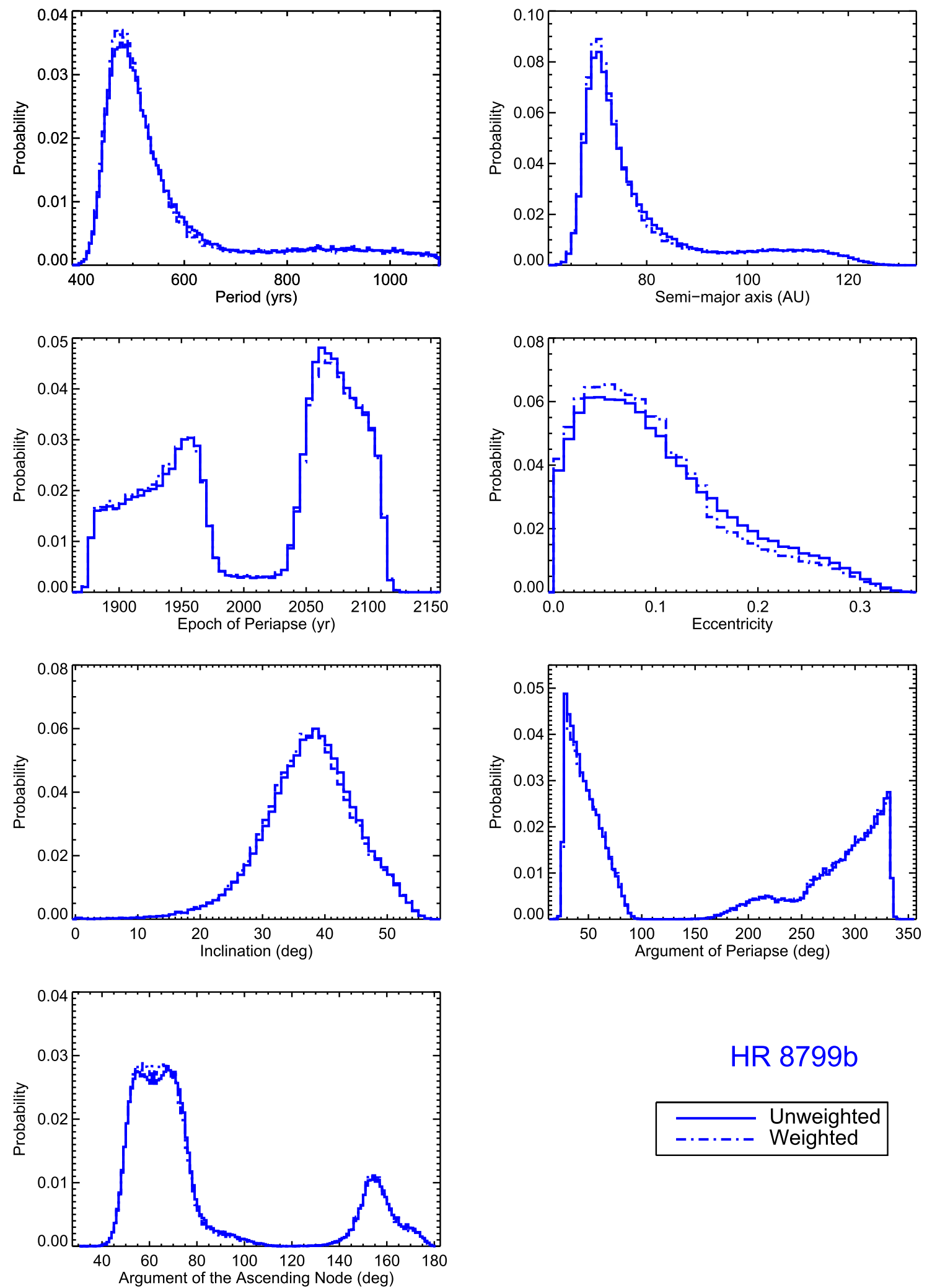

HR 8799b

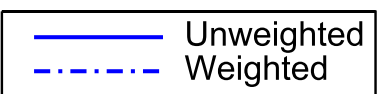

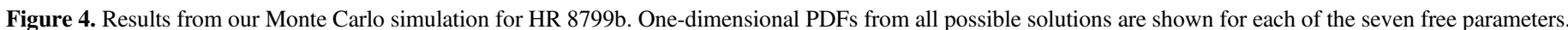

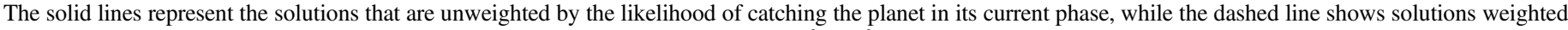
by this likelihood. Low-eccentricity solutions are favored, as is an inclination of $38^{\circ} \pm 7^{\circ}$.

astrometry by using the same camera and reduction techniques. The small reduced $\chi^{2}$ and low fit residuals suggest that our error bars at least properly capture the remaining systematic uncertainties.
For the most part, the orbital parameters we derive are consistent with previous fits to within the uncertainties. We do find a slight difference between our preferred inclinations and those in previous works, with ours favoring values closer to 

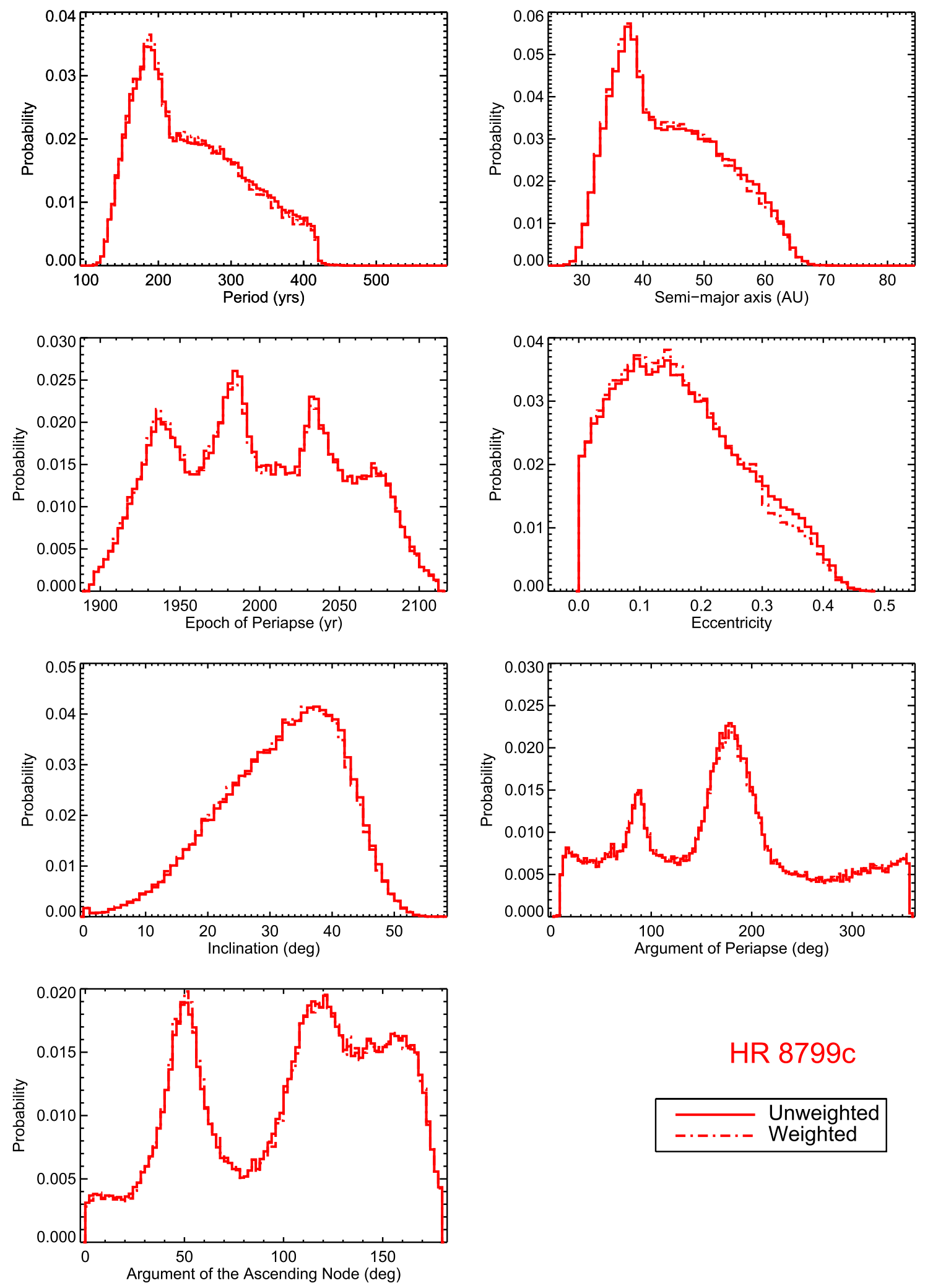

\section{HR 8799c}

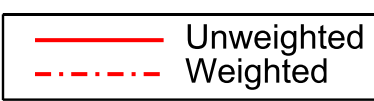

Figure 5. Results from our Monte Carlo simulations for HR 8799c. One-dimensional PDFs from all possible solutions are shown for each of the seven free parameters. Solid lines represent unweighted solutions while dashed lines show weighted solutions. All allowed eccentricities have a value of $<0.5$, and the preferred inclination is $37^{\circ} \pm 12^{\circ}$.

$\sim 30^{\circ}-40^{\circ}$ than to $10^{\circ}-30^{\circ}$ (e.g., Currie et al. 2012 ; Esposito et al. 2013; Pueyo et al. 2015). Still, for all four planets, inclinations of $\lesssim 30^{\circ}$ are allowed, consistent with a number of previous works that assumed a value of $28^{\circ}$ for the system inclination. It is notable, however, that a few analyses unrelated to orbit fitting predict disparate inclinations for this system. Results from asteroseismology suggest that an inclination of $>40^{\circ}$ is preferred (Wright et al. 2011) for the star HR 8799 

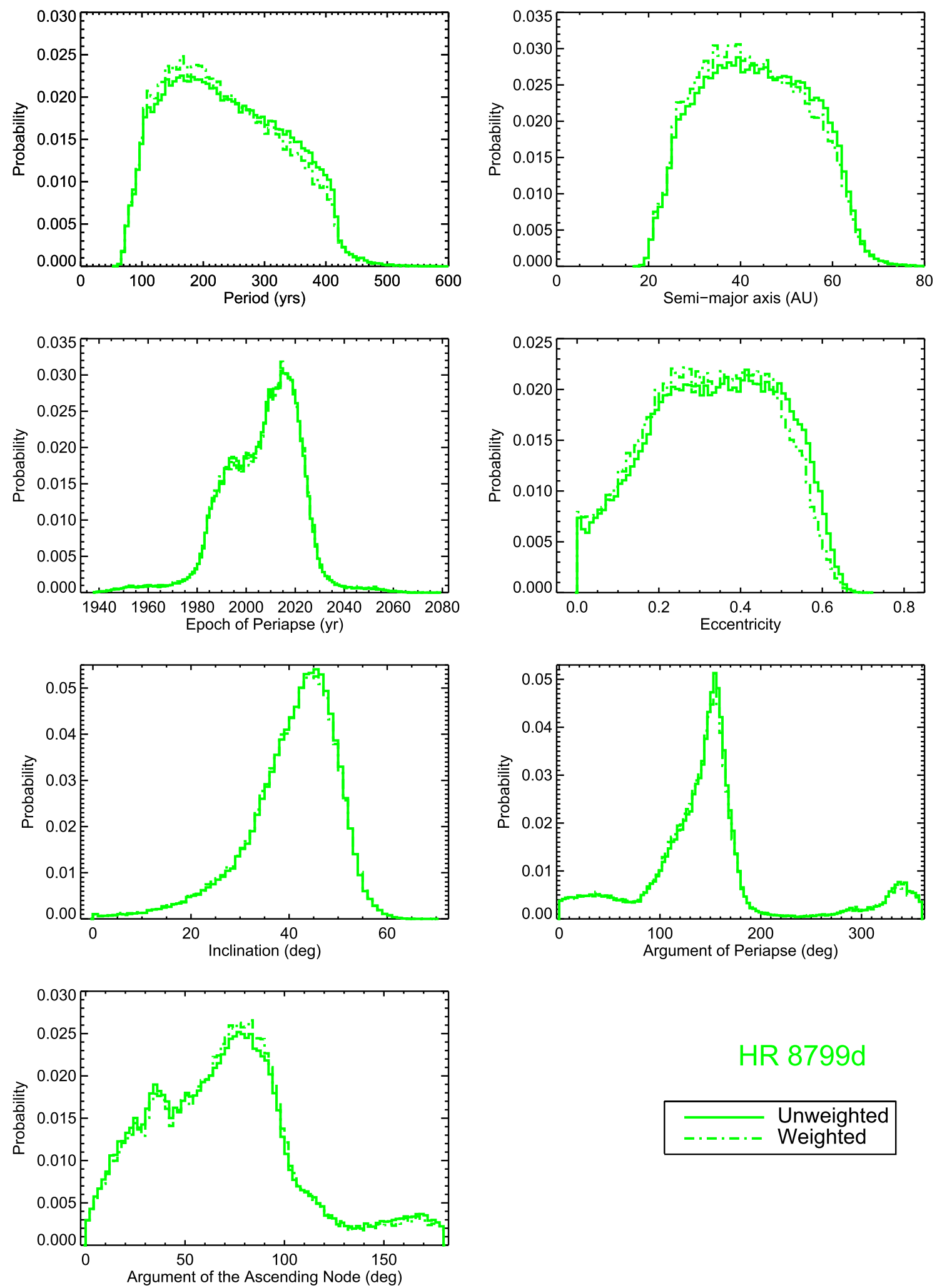

\section{HR 8799d}

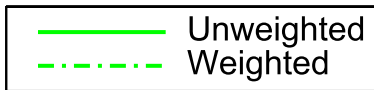

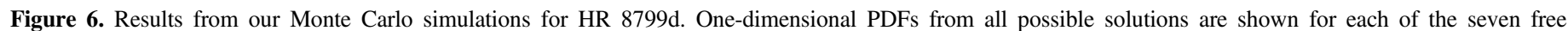

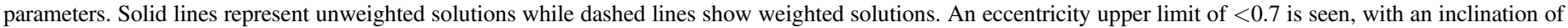
$45^{\circ} \pm 8^{\circ}$. While this inclination is slightly offset from HR $8799 \mathrm{~b}$ and $\mathrm{c}$, it is consistent to within the uncertainties.

itself. Meanwhile, analysis of the far-infrared emissions from the debris disks in the HR 8799 system suggest an inclination of $<25^{\circ}$ (Su et al. 2009), with a recent analysis from Matthews et al. (2014) suggesting a disk inclination of $26^{\circ} \pm 3^{\circ}$.
Most recently, ALMA observations resolved the HR 8799 planetessimal belt, and found an inclination of $40_{-6}^{+5 \circ}$ (Booth et al. 2016). Our analysis gives an inclination more consistent with the asteroseismology and the ALMA results than 

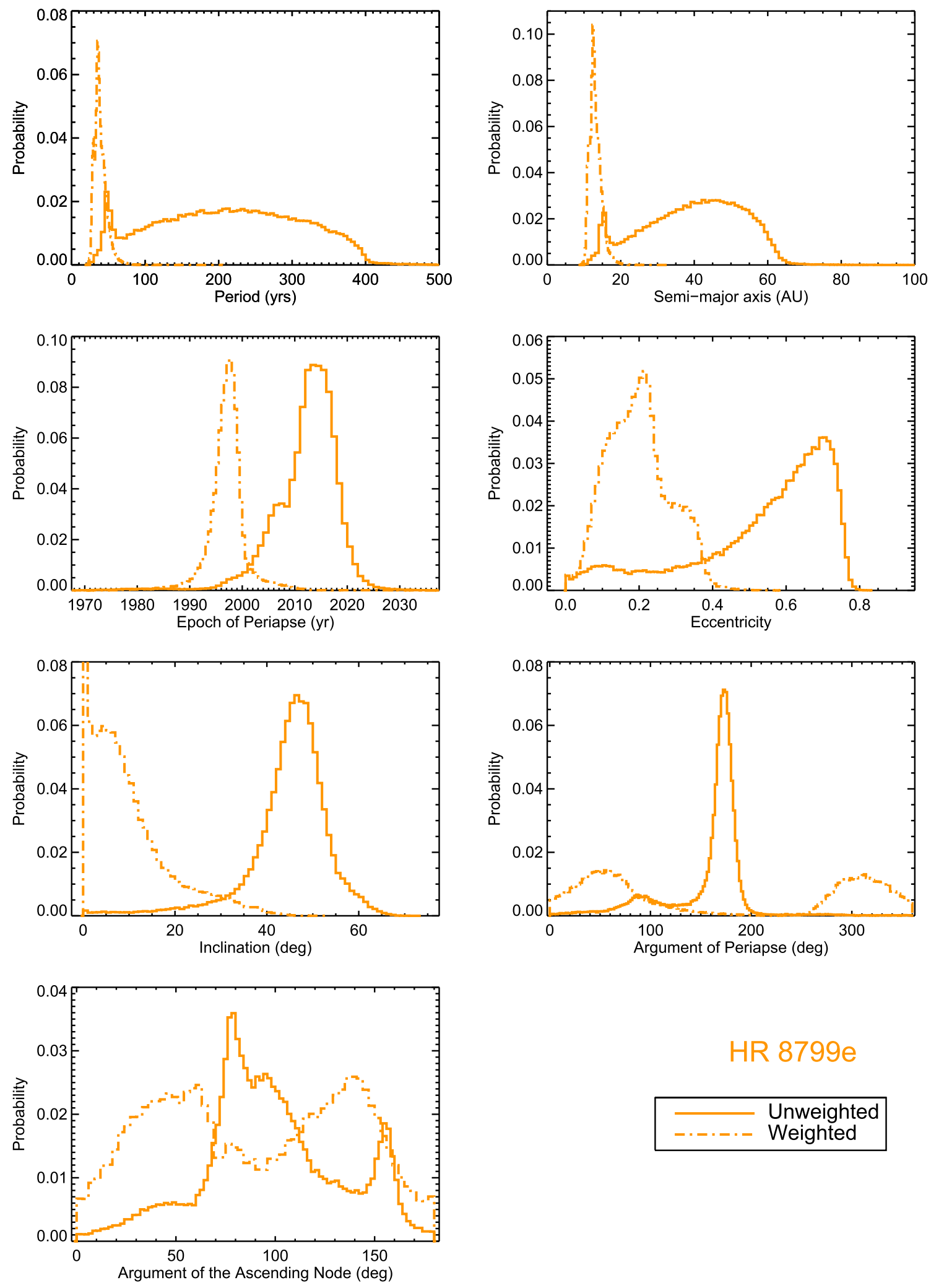

\section{HR 8799e}

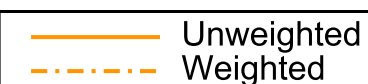

Figure 7. Results from our Monte Carlo simulations for HR 8799e. One-dimensional PDFs from all possible solutions are shown for each of the seven free parameters. The solid lines represent the solutions unweighted by the likelihood of catching the planet in its current phase, while the dashed line shows solutions weighted by this likelihood. High eccentricities are favored in the unweighted case, along with periastron passage close to the current epoch. The inclination also shifts from about $50^{\circ}$ in the unweighted case to preferring face on in the weighted case. Because of the higher astrometric uncertainties, our constraints on the orbital parameters of this planets are still quite limited. 

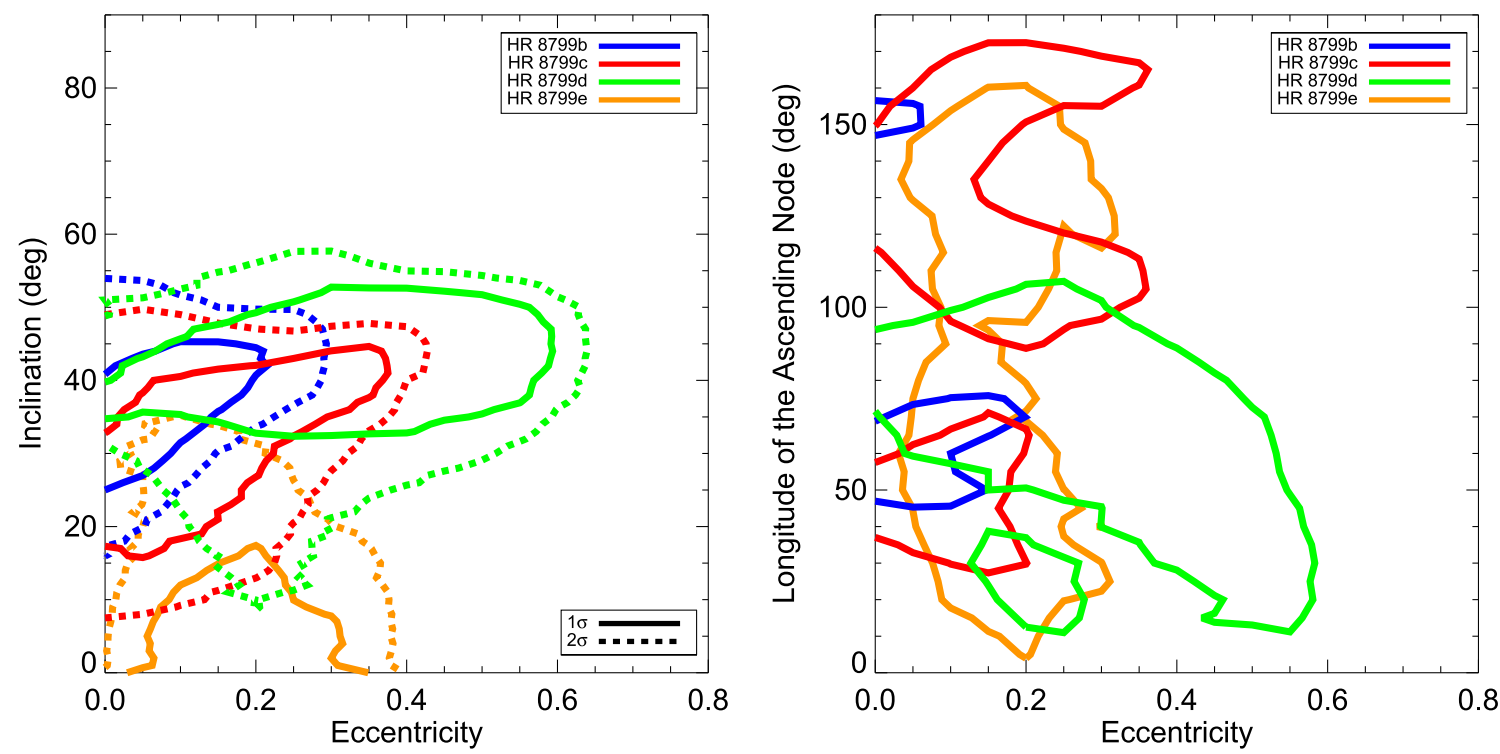

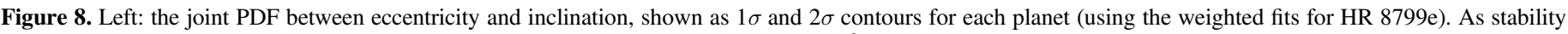

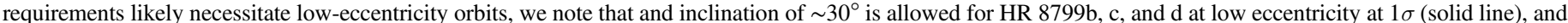

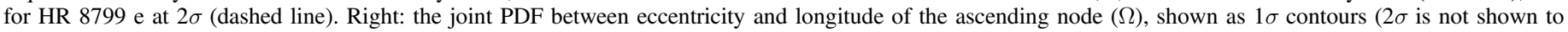
enhance clarity). At low eccentricity, all planets are consistent between $50^{\circ}$ and $70^{\circ}$.

previous work, offering the interesting possibility that the star, disk, and planets are in fact co-aligned. However, our analysis is also consistent within $1 \sigma$ with the results from far-infrared data. Given the large range of possible inclinations in this and in previous works, we believe it is premature to make claims about possible mutual inclinations between the star, planets, and debris disks. It is comforting, however, that all analyses suggest non-face-on configurations for the system.

While no statistically significant mutual inclinations between any of the planets is found, especially when orbits that do not cross each other's Hill radii are considered, we are interested in assessing the resulting orbital parameters if the planets were forced to be coplanar. This is similar to previous works in which coplanarity was assumed based on dynamical arguments (e.g., Soummer et al. 2011; Goździewski \& Migaszewski 2014). We opted to use values of inclination and $\Omega$ sampled from the solutions for HR $8799 \mathrm{~b}$ shown in Figure 4. From these distributions, we randomly selected 10,000 values for inclination and $\Omega$ and then performed a similar Monte Carlo simulation to those described above for HR 8799c, d, and e, fixing those values (weighting the fits for HR 8799e). The results of these simulations are shown in Figure 10. All fits return reasonable values of reduced $\chi^{2}$, with the best fit reduced $\chi^{2}$ of $0.32,0.58$, and 0.46 for HR $8799 \mathrm{c}, \mathrm{d}$, and e, respectively. These values are very close to our overall best fit $\chi^{2}$. The solutions also tend to yield relatively low eccentricities, with the upper limit for eccentricity dropping to 0.3 for HR 8799 c and d, and 0.5 for HR $8799 \mathrm{e}$. This is another indication that coplanar solutions for all the planets in HR 8799are fully consistent with current astrometry. Given the distributions of allowed inclinations in our various orbit fits, we believe that no offset is detectable with current astrometry and phase coverage if it is $\lesssim 20^{\circ}$-offsets larger than this would be detectable.

Previous dynamical analysis has also suggested that the planets may orbit in either a 1:2:4:8 resonance configuration (e.g., Goździewski \& Migaszewski 2014) or a 1:2:4 resonance between the inner planets with HR 8799b not participating in any resonance (e.g., Fabrycky \& MurrayClay 2010; Marois et al. 2010b). In Figures 9 and 10 we note the locations of $1: 2: 4: 8$ period ratios assuming that the peak and $1 \sigma$ values of the period distribution of HR $8799 \mathrm{e}$ is the starting point of the chain. In both cases, the distributions for all planets are consistent with these ratios within $1 \sigma$. In the case of the constraints from non-crossing Hill radii, the peak of the distribution for $\mathrm{HR} 8799 \mathrm{~b}$ is only marginally consistent with this resonance chain. Still, both sets of analysis return results consistent with dynamical modeling predictions.

In order to further assess the orbital parameters required for the planets, particularly HR $8799 \mathrm{~b}$, to be consistent with a $1: 2: 4: 8$ resonance in the case of non-crossing orbits, we performed another simulation where we used the distribution of periods from HR 8799e shown in Figure 9, fixed a period with the appropriate multiplicative factor for the other three planets, and then determined the preferred orbital solution. This analysis is similar to that described above where the plane of the orbit was fixed for three of the planets. The results of this simulation are shown in Figure 11. In order to get fits that satisfy this resonance, solutions with very specific values of $T_{o}$ and $\omega$ are required that lead to higher eccentricity values than in our other simulations. However, the orbital plane parameters remain consistent with previous simulations, and low-eccentricity solutions are still allowed. There is also a tendency for the solutions for HR 8799d to be slightly higher eccentricity than in the case of the previous non-crossing solutions, but again lower eccentricity solutions are allowed. Future dynamical stability simulations that further consider the case where HR 8799b does not participate in a resonance with the inner three planets could potentially yield interesting additional constraints on its orbital configuration. 

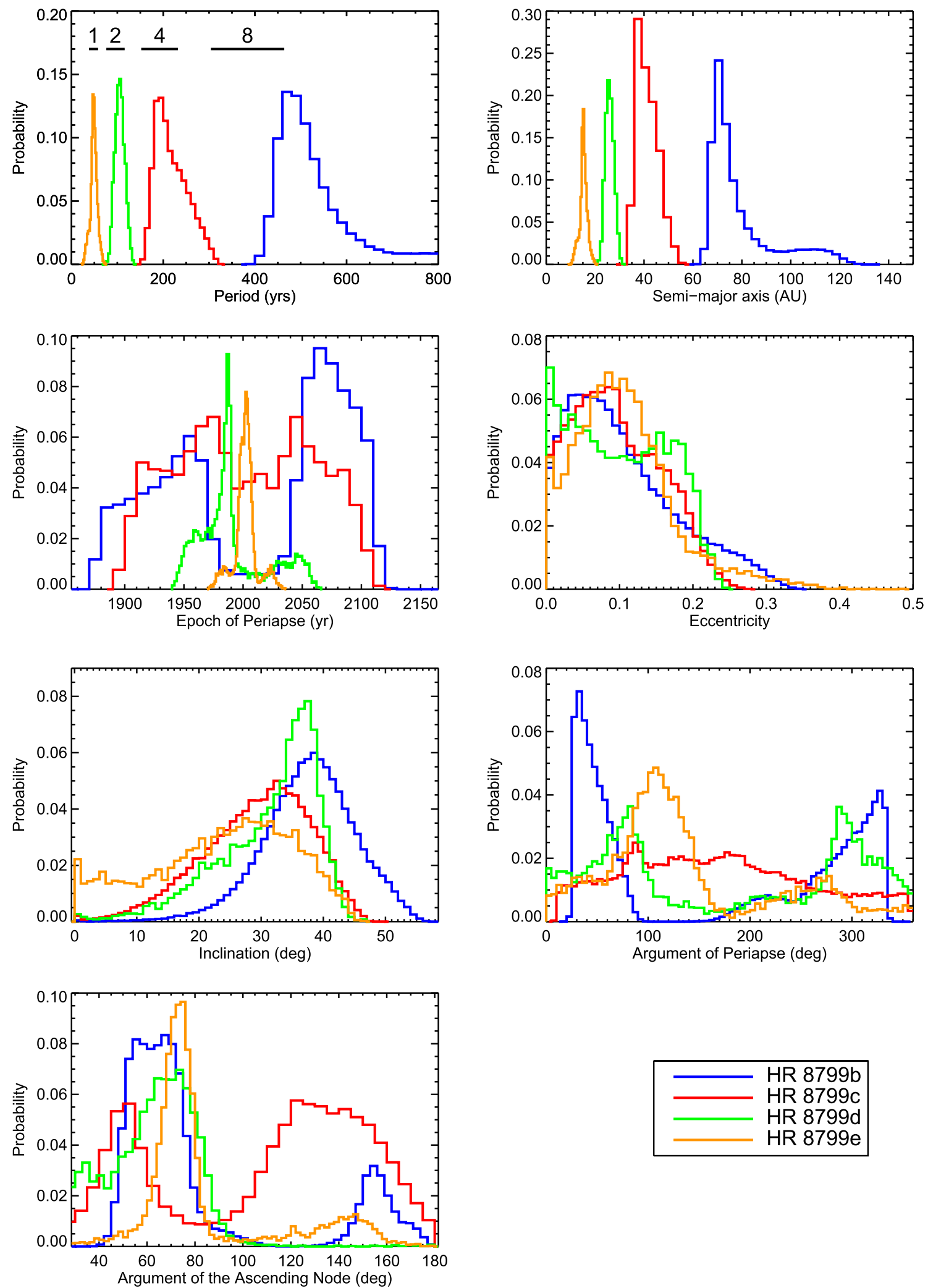

Figure 9. One-dimesonsional PDFs for all four planets for only those orbits that do not come within the Hill radius of each other. The solutions for HR 8799e are drawn from weighted simulations. Eccentricity upper limits are $<0.3$ for all planets, and orbital plane distributions remain fully consistent, with strong overlap in both inclination and $\Omega$. Also noted is the locations of 1:2:4:8 period ratios assuming the peak and $1 \sigma$ values of the distribution for HR $8799 \mathrm{e}$ is the start of the chain. In this case, all four planets are consistent with these ratios to within $1 \sigma$. 

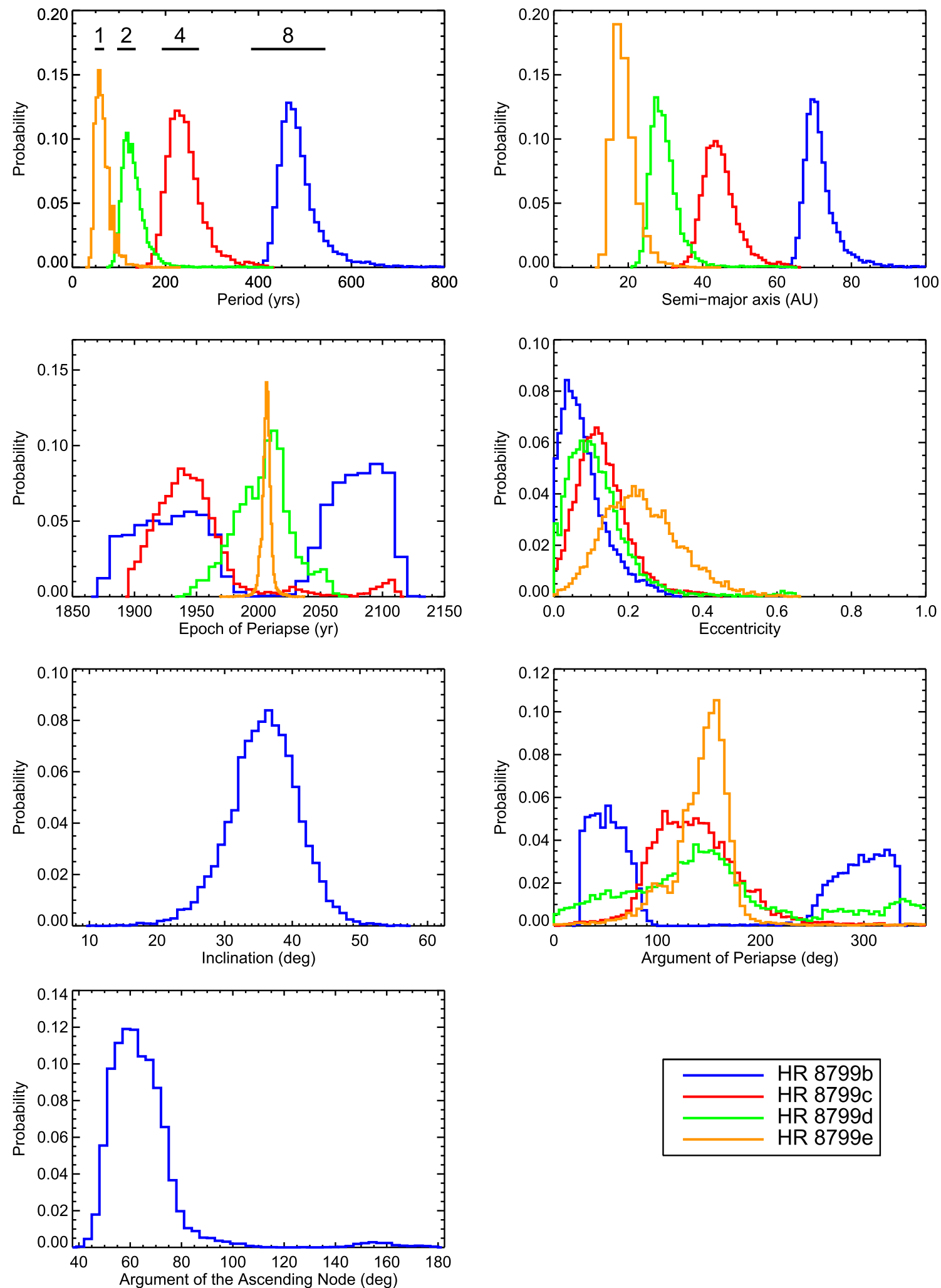

Figure 10. One-dimesonsional PDFs for all four planets under the assumption that they are coplanar. Inclination and $\Omega$ are sampled from 10,000 randomly selected solutions for HR 8799b in our larger Monte Carlo simulation. Assuming coplanarity with HR 8799b leads to eccentricity upper limits of 0.3 for HR $8799 \mathrm{c}$ and d, and 0.5 for HR $8799 \mathrm{e}$. The reduced $\chi^{2}$ for these fits for HR $8799 \mathrm{c}, \mathrm{d}$, and e are small, showing that coplanarity is completely consistent with our data. Also, as in Figure 9, we note the locations of 1:2:4:8 period ratios assuming the distribution for HR 8799e is the start of the chain. All four planets align very well with these period ratios. 

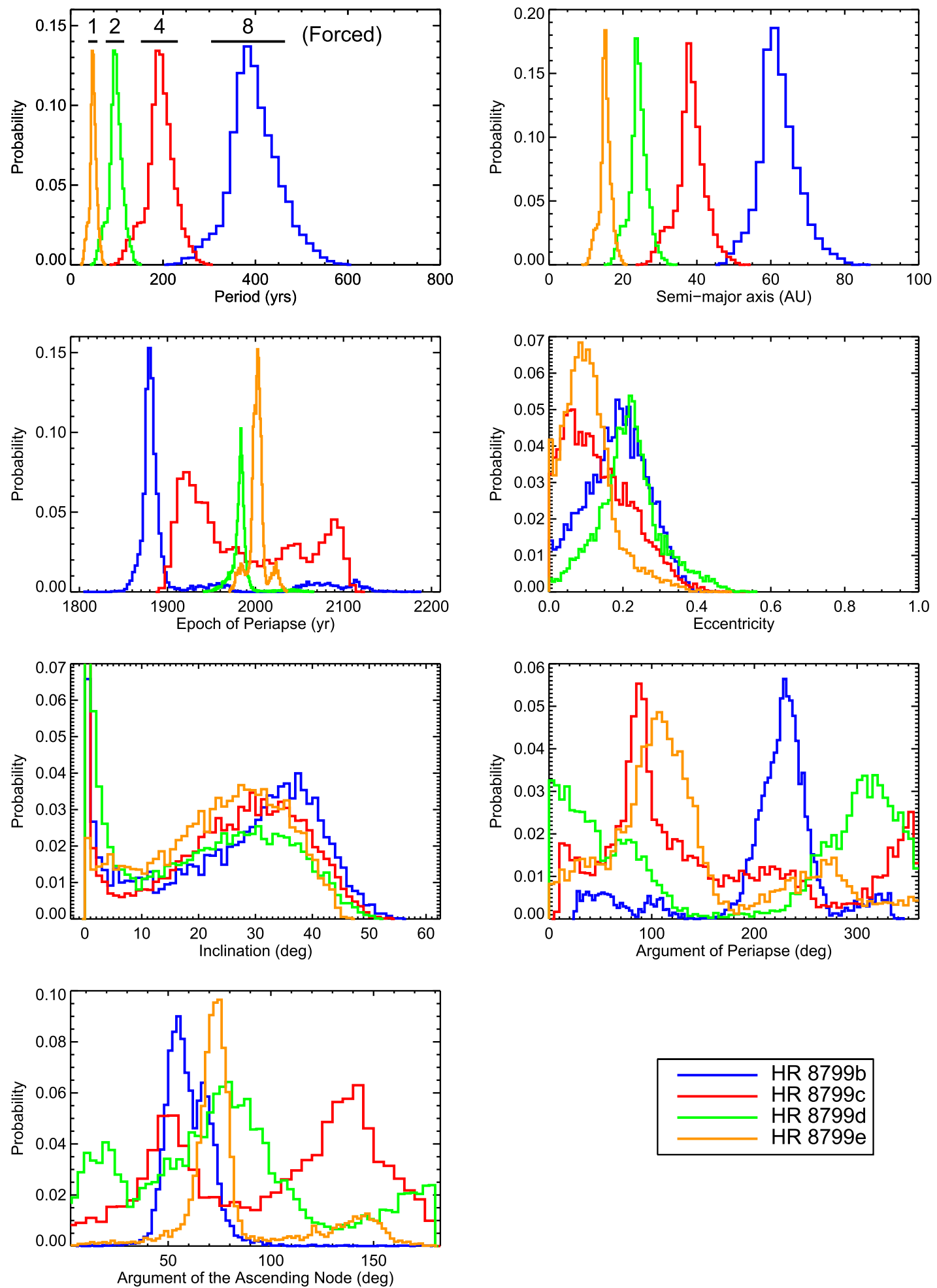

Figure 11. One-dimensional PDFs for all four planets under the assumption that the periods are in a 1:2:4:8 resonance. The periods are randomly sampled from 10,000 solutions for HR 8799e in the case where the orbits are non-crossing (Figure 9). The periods are then multipled by the appropriate factor for each planet. There is also generally a fairly fixed set of orbital parameters that fit this criteria for HR 8799b, with strong weighting toward specific values for $T_{o}$ and $\omega$. In addition, resonance is forced, and higher eccentricity solutions are preferred for HR 8799b and HR 8799d than we find in our other simulations.

\subsection{Impact of Biases on Orbital Parameters}

For undersampled orbits such as these and for other directly imaged exoplanets, it is important to assess whether systematics in astrometric data sets play a role in the output of orbit fitting, regardless of the method of the fitting. For instance, poor $\chi^{2}$ values when minimal data points exist, sampling less than $5 \%$ of an orbital period, suggests that data points and error bars may need to be reassessed before conclusions are drawn about the orbital parameters. For instance, in this work we have shown that using a data set in which systematics are controlled leads to slight differences in the predicted orbital elements 
compared to previous work. We find that the inclination of HR 8799 d is not inconsistent with the other planets, in contrast to previous work using all available astrometry (Currie et al. 2012; Pueyo et al. 2015). In order to determine whether systematics could generate an apparent shift in the distribution of orbital inclinations, we performed a short set of simulations using an assumed orbit for HR 8799d. This orbit had an eccentricity of 0.02 , an inclination of $29^{\circ}$, and $\Omega$ of $59^{\circ}$. We generated a simulated data set based on this orbit sampled at the same times as all previous astrometric measurements for HR 8799d. We then estimated the possible size of systematic offsets between data sets based on the offsets of data points from orbit fits in Soummer et al. (2011), Esposito et al. (2013), Pueyo et al. (2015), and Zurlo et al. (2015). The apparent size of these offsets range from 5 to 30 mas. We then applied offsets randomly sampled from this size range to different data points in our simulated astrometric data. Our goal was not to encompass all possible systematics, but rather to construct a notional representation of a possible data set and see if we could generate a significant inclination offset from the true inclination. We find that applying systematics of this magnitude to the data can result in the most likely inclination of the planet being higher than the true value by $5^{\circ}-10^{\circ}$. However, the distribution always encompasses the correct value of $29^{\circ}$ such that it would be allowed to $1-2 \sigma$. This is somewhat analogous to the results from Pueyo et al. (2015), where there is overlap in the inclination distributions of all four planets at slightly greater than $1 \sigma$, but the maximum likelihood values are off by $\sim 15^{\circ}$. Thus, it is important to account for possible systematics such as those introduced from using astrometry from multiple cameras and data pipelines in the case where the orbits sample $\lesssim 10 \%$ of the total period.

\section{SUMMARY}

We have presented both new and updated astrometric measurements from Keck for the planets around HR 8799. In order to minimize systematics, we have performed orbit fits to astrometry from Keck and NIRC2 only, and shown that the orbital planes of the planets are consistent based on current data. We have also shown that the eccentricities are likely low, and that at least the inner three planets have period distributions consistent with a 1:2:4 resonance configuration. It is important to interpret the results of orbital fits to long-period systems with minimal phase coverage with some caution, as systematics can bias the resulting parameter distributions.

Future work will include an update to dynamical models for the system using our improved astrometric measurements, and continued monitoring of the system to promote additional acceleration detections. The data from newly commissioned instruments like the Gemini Planet Imager and SPHERE have been shown to yield improved astrometric error bars compared to earlier work (e.g., De Rosa et al. 2015; Zurlo et al. 2015), and if properly calibrated for systematics, have the potential to yield stronger constraints on the orbital parameters of this fascinating multiplanet system. Our continued monitoring of the system with Keck and $\mathrm{NIRC2}$, with their exquisite astrometric calibration, will play a vital role in constraining biases in future observations with other facilities.

The authors thank observing assistants Joel Aycock, Heather Hershley, Carolyn Jordan, Gary Puniwai, Julie Rivera, Terry Stickel, and Cynthia Wilburn, and supporting astronomers
Randy Campbell, Al Conrad, Scott Dahm, Grant Hill, Mark Kassis, Jim Lyke, Luca Rizzi, and Hien Tran, for their help in obtaining the observations. We thank Eric Nielsen, Tuan Do, and Jessica $\mathrm{Lu}$ for helpful conversations about fitting methodology. We also thank Sylvana Yelda for helpful suggestions (7439). We also thank an anonymous referee for comments on this manuscript. Portions of this work were performed under the auspices of the U.S. Department of Energy by Lawrence Livermore National Laboratory under Contract DE-AC52-07NA27344. This research was supported by a NASA Origins of Solar Systems grant to LLNL. Portions of this work were also performed at the Dunlap Institute for Astronomy \& Astrophysics, University of Toronto. The Dunlap Institute is funded through an endowment established by the David Dunlap family and the University of Toronto. The W.M. Keck Observatory is operated as a scientific partnership among the California Institute of Technology, the University of California, and the National Aeronautics and Space Administration. The Observatory was made possible by the generous financial support of the W.M. Keck Foundation. The authors also wish to recognize and acknowledge the very significant cultural role and reverence that the summit of Maunakea has always had within the indigenous Hawaiian community. We are most fortunate to have the opportunity to conduct observations from this mountain.

\section{REFERENCES}

Amara, A., \& Quanz, S. P. 2012, MNRAS, 427, 948

Baines, E. K., White, R. J., Huber, D., et al. 2012, ApJ, 761, 57

Bergfors, C., Brandner, W., Janson, M., Köhler, R., \& Henning, T. 2011, A\&A, 528, A134

Booth, M., Jordán, A., Casassus, S., et al. 2016, MNRAS, 460, L10 Chauvin, G., Lagrange, A.-M., Beust, H., et al. 2012, A\&A, 542, A41

Crida, A., Masset, F., \& Morbidelli, A. 2009, ApJL, 705, L148

Currie, T., Burrows, A., Girard, J. H., et al. 2014, ApJ, 795, 133

Currie, T., Fukagawa, M., Thalmann, C., Matsumura, S., \& Plavchan, P. 2012 ApJL, 755, L34

De Rosa, R. J., Nielsen, E. L., Blunt, S. C., et al. 2015, ApJL, 814, L3

Duchêne, G., Beust, H., Adjali, F., Konopacky, Q. M., \& Ghez, A. M. 2006, A\&A, 457, L9

Esposito, S., Mesa, D., Skemer, A., et al. 2013, A\&A, 549, A52

Fabrycky, D. C., \& Murray-Clay, R. A. 2010, ApJ, 710, 1408

Fergus, R., Hogg, D. W., Oppenheimer, R., Brenner, D., \& Pueyo, L. 2014, ApJ, 794, 161

Fukagawa, M., Itoh, Y., Tamura, M., et al. 2009, ApJL, 696, L1

Galicher, R., \& Marois, C. 2011, in Second Int. Conf. on Adaptive Optics for Extremely Large Telescopes Online at http://ao4elt2.lesia.obspm.fr

Ghez, A. M., Salim, S., Weinberg, N. N., et al. 2008, ApJ, 689, 1044

Gomez Gonzalez, C. A., Absil, O., Absil, P.-A., et al. 2016, arXiv:1602.08381

Goździewski, K., \& Migaszewski, C. 2014, MNRAS, 440, 3140

Hinz, P. M., Rodigas, T. J., Kenworthy, M. A., et al. 2010, ApJ, 716, 417

Høg, E., Fabricius, C., Makarov, V. V., et al. 2000, A\&A, 355, L27

Ingraham, P., Marley, M. S., Saumon, D., et al. 2014, ApJL, 794, L15

Kalas, P., Graham, J. R., Chiang, E., et al. 2008, Sci, 322, 1345

Kuzuhara, M., Tamura, M., Kudo, T., et al. 2013, ApJ, 774, 11

Lafrenière, D., Marois, C., Doyon, R., \& Barman, T. 2009, ApJL, 694, L148

Lafrenière, D., Marois, C., Doyon, R., Nadeau, D., \& Artigau, É. 2007, ApJ, 660, 770

Lagrange, A.-M., Gratadour, D., Chauvin, G., et al. 2009, A\&A, 493, L21

Lu, J. R., Ghez, A. M., Hornstein, S. D., et al. 2009, ApJ, 690, 1463

Macintosh, B., Graham, J. R., Barman, T., et al. 2015, Sci, 350, 64

Maire, A.-L., Skemer, A. J., Hinz, P. M., et al. 2015, A\&A, 576, A133

Marois, C., Correia, C., Galicher, R., et al. 2014, Proc. SPIE, 9148, 91480U

Marois, C., Lafrenière, D., Doyon, R., Macintosh, B., \& Nadeau, D. 2006, ApJ, 641, 556

Marois, C., Macintosh, B., Barman, T., et al. 2008, Sci, 322, 1348

Marois, C., Macintosh, B., \& Véran, J.-P. 2010a, Proc. SPIE, 7736, 77361J

Marois, C., Zuckerman, B., Konopacky, Q. M., Macintosh, B., \& Barman, T. 2010b, Natur, 468, 1080 
Matthews, B., Kennedy, G., Sibthorpe, B., et al. 2014, ApJ, 780, 97

Meshkat, T., Kenworthy, M. A., Quanz, S. P., \& Amara, A. 2014, ApJ, 780, 17

Metchev, S., Marois, C., \& Zuckerman, B. 2009, ApJL, 705, L204

Millar-Blanchaer, M. A., Graham, J. R., Pueyo, L., et al. 2015, ApJ, 811,18

Moro-Martín, A., Rieke, G. H., \& Su, K. Y. L. 2010, ApJL, 721, L199

Pueyo, L., Soummer, R., Hoffmann, J., et al. 2015, ApJ, 803, 31

Rajan, A., Barman, T., Soummer, R., et al. 2015, ApJL, 809, L33

Rameau, J., Chauvin, G., Lagrange, A.-M., et al. 2013, ApJL, 772, L15

Reidemeister, M., Krivov, A. V., Schmidt, T. O. B., et al. 2009, A\&A, 503,247

Serabyn, E., Mawet, D., \& Burruss, R. 2010, Natur, 464, 1018
Skemer, A. J., Hinz, P. M., Esposito, S., et al. 2012, ApJ, 753, 14

Soummer, R., Brendan Hagan, J., Pueyo, L., et al. 2011, ApJ, 741, 55

Soummer, R., Pueyo, L., \& Larkin, J. 2012, ApJL, 755, L28

Su, K. Y. L., Rieke, G. H., Stapelfeldt, K. R., et al. 2009, ApJ, 705, 314

Sudol, J. J., \& Haghighipour, N. 2012, ApJ, 755, 38

van Leeuwen, F. 2007, A\&A, 474, 653

Veras, D., Crepp, J. R., \& Ford, E. B. 2009, ApJ, 696, 1600

Wizinowich, P. L., Chin, J., Johansson, E., et al. 2006, Proc. SPIE, 6272, 9

Wright, D. J., Chené, A.-N., De Cat, P., et al. 2011, ApJL, 728, L20

Yelda, S., Lu, J. R., Ghez, A. M., et al. 2010, ApJ, 725, 331

Zuckerman, B., Rhee, J. H., Song, I., \& Bessell, M. S. 2011, ApJ, 732, 61

Zurlo, A., Vigan, A., Galicher, R., et al. 2015, arXiv:1511.04083 